\title{
Accurate Control of AMT Gear Shifting Based on Fuzzy Adaptation
}

\author{
Yulong Lei, Yuchen Zhang, Yao Fu' ${ }^{a}$, Bo Yan ${ }^{b}$ \\ Jilin University, State Key Laboratory of Automobile Simulation and Control, Changchun 130022; \\ afu_yao@jlu.edu.cn,b18843102529@163.com
}

Keywords: PID control, shifting actuator, control strategy

\begin{abstract}
For shorting the shifting time and improving the vehicle acceleration, it is necessary to develop an excellent control strategy of electing and shifting. In this paper we developed the strategy of the electric shifting actuators based on fuzzy PID control and established a fuzzy dual closed-loop PID controller model to simulate its control effect by using the Matlab/Simulink software. From the simulation results, we get the conclusions: the designed controller of shifting actuator has a fast response speed, high control accuracy, and good stability.
\end{abstract}

\section{Introduction}

AMT is added with electronic control actuator and operating system on the base of traditional manual mechanical transmission[1,2], using micro electronic technology, driving and control theory, and taking electronic controller unit (ECU) as the core, operating shift actuator, clutch and accelerator by electric, hydraulic or pneumatic actuators to realize the automatic starting and shifting. The biggest advantage of AMT transmission is that it gets rid of the working mode of traditional automatic transmission, and the complex planetary gear set and the torque converter is no longer needed anymore. So the transmission efficiency and speed performance can be improved sharply.

\section{Operating principle of the electric control and electric shifting actuator}

The working principle of the gear shifting actuator is introduced in this paper, using 1-N-2 actuator of the AMT system as an example, as is shown in Fig1. When AMT needs to shift, the TCU controls the shifting motor to rotate, and drive the screw connected with the motor shaft to rotate, so that the balls of the ball screw move along the screw axis, which drives the rocker arm connected with the ball to rotate around its axis. From Fig1, it can be saw that the end of the rocker arm has a contact with the internal gear shifting fork of the transmission. Rotation of the rocker arm can push the shift fork to move in the axial direction, and drive the meshing sleeve to move, to achieve the pick and hang. TCU collects the real-time angular displacement of the rocker rotation by the angular displacement sensor, and calculates the real-time axial displacement of current meshing sleeve by the geometric relationship, to determine whether the shift is in place. And it can achieve the closed-loop control of the shifting motor through the feedback of the signal, and improve the control accuracy of the shift and shifting quality of the AMT system. 


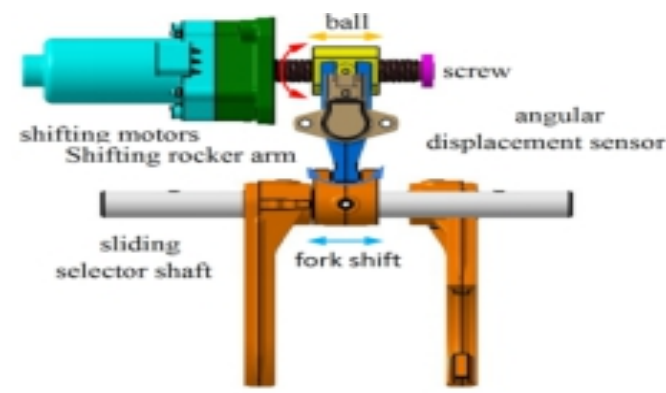

Figure 1. Principle of process diagram of electric control and electric shifting actuator

\section{The strategy research of the electric shifting actuators based on fuzzy PID control}

Fuzzy controller has strong robustness and good dynamic response characteristic, but it is difficult to eliminate the static error; PID controller has a very high precision while its dynamic response is very poor. In this paper, the advantages of both are combined to adjust the three control parameters of the position of the PID controller in real time by fuzzy reasoning. Besides the controller based on fuzzy PID of electric shifting actuator is developed, to guarantee that the electric shifting actuator controller not only has high precision, but also has strong adaptive ability and robustness. The fuzzy control system mainly consists of fuzzificationn fuzzy inference and defuzzification[4,5]

\section{Fuzzy PID controller}

The computed sampling error and its rate of change are taken as the inputs and the fuzzy rule tables of scaling factor 、 integral coefficient and differential coefficient are established by means of fuzzy reasoning in the fuzzy PID controller. The fuzzy PID controller also can adjust scaling factor integral coefficient and the differential coefficient automatically. Not only the good fuzzy control characteristic of adaptive ability and robustness can be given play to, but also the control accuracy can be effectively improved in the fuzzy PID controller[6,7]. The schematic diagram of a fuzzy PID controller is shown in Fig.2.

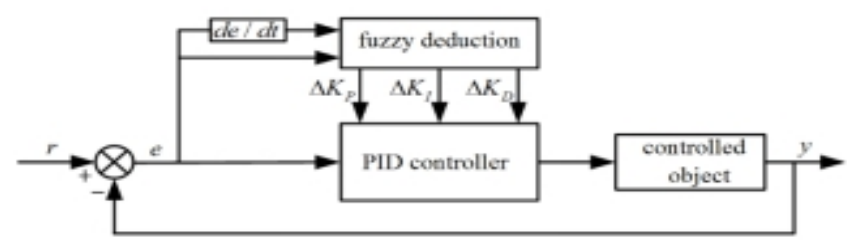

Figure 2. Schematic diagram of fuzzy PID controller

As is shown in Fig.2 fuzzy PID controller consists of fuzzy reasoning and PID controller. The sampling error e and its rate of change de/dt are taken as the inputs in the fuzzy reasoning module, and the variation 、 and corresponding to scaling factor vintegral coefficient and differential coefficient of the PID controller are exported. The fuzzy PID controller does real-time detection of the error and its rate of change in the process of control, and adjusts the value of and in real time. The value of the above parameter is given by:

$$
\begin{aligned}
& \mathrm{K}_{\mathrm{P}}=\mathrm{K}_{\mathrm{P} 0}+\Delta \mathrm{K}_{\mathrm{P}} \\
& \mathrm{K}_{\mathrm{I}}=\mathrm{K}_{\mathrm{I} 0}+\Delta \mathrm{K}_{\mathrm{I}} \\
& \mathrm{K}_{\mathrm{D}}=\mathrm{K}_{\mathrm{D} 0}+\Delta \mathrm{K}_{\mathrm{D}}
\end{aligned}
$$

In the Eq. 1, and are the initial values of scaling factor integral coefficient and differential coefficient respectively. 


\section{The developing of the shifting actuator controller based on fuzzy self-adaptation and double-closed-loop PID control strategy}

In this paper the electric shift actuator is the controlled object and the fuzzy control rule is introduced into the closed-loop PID controller of the direct current shifting motor for its disturbance rejection and adaptive ability in order to improve the control precision of the electric shift actuators and shift quality of the pure electric bus. The schematic diagram of the electric shifting actuator controller which is based on fuzzy self-adaptation and double-closed-loop PID control strategy is shown in Fig3.

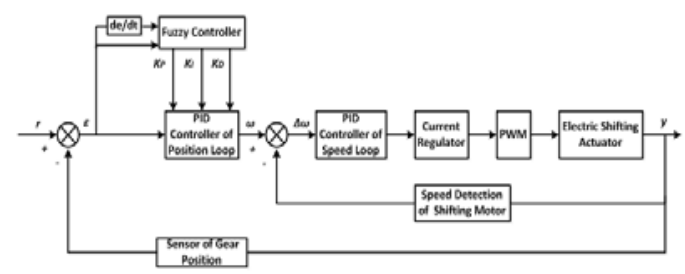

Figure 3. The schematic diagram of the electric shifting actuator controller based on fuzzy self-adaptation and double-closed-loop PID control strategy

The position and speed double-closed-loop control is used in electric shifting actuator controller, the out loop is the position closed loop and the inside one is the current closed loop. The feedback element of the position loop PID controller is a gear position sensor installed on the shifting actuator, its input is position deviation e of target position and current position collected by the sensor and the output is the target control speed of motor which is the setting speed of speed loop. The input of the speed loop PID controller is deviation of the set speed and feedback value, its feedback value is calculated by the feedback value of the motor encoder, and the output is the setting current of the current regulator.

The three control parameters and of the position loop PID control corresponding to the collected and calculated positional deviation e and its rate of change can be updated in real time in the fuzzy controller. So the adaptive ability and robustness of PID controller can be improved on the basis of ensuring the control precision. We will focus on fuzzy controller in the flowing of this article.

\section{The membership function between the inputs and the outputs}

The fuzzy subset defining the variables above is, and the variables $\mathrm{E}$ and are all divided into 7 grade whose subordinating degree functions are all triangular function, as is shown in Fig. 4.

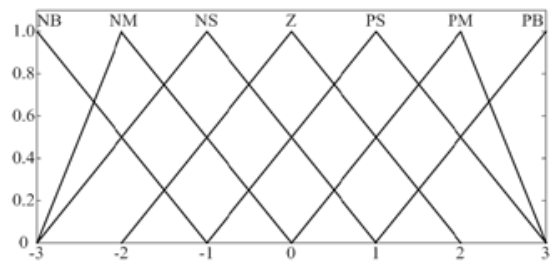

Figure 4. Membership functions of input and output variables

\section{The fuzzy control rule}

The fuzzy rule follows the following ${ }^{[8]}$ : 
1.When the gear's positional deviation $|\mathrm{E}|$ is very big, a bigger scaling factor should be chosen for the purpose of improving the response speed of the control system; to avoid the overshoot of the system, the value of the integral coefficient should be 0 ;

2. When the value of the gear's positional deviation $|\mathrm{E}|$ and its rate of change $|\mathrm{EC}|$ is medium, a smaller scaling factor should be chosen to avoid the overshoot of the system; for the purpose of improving the response speed of the system, the medium integral coefficient and differential coefficient should be chosen.

3.The value of the $|\mathrm{EC}|$ reflects the rate of change of the gear's positional deviation, when the value of $|\mathrm{EC}|$ is very big, the value of scaling factor should be decreased properly while the value of the integral coefficient should be increased properly.

The established fuzzy control-rule table is shown in the following 3 tables.

Table 1

\begin{tabular}{|l|l|l|l|l|l|l|l|}
\hline \multirow{2}{*}{ E } & \multicolumn{7}{l|}{ EC } \\
\cline { 2 - 8 } & N & N & N & Z & PS & P & P \\
& B & M & S & & & M & B \\
\hline N & Z & Z & N & N & N & N & N \\
B & & & M & M & M & B & B \\
\hline N & P & Z & N & N & N & N & N \\
M & S & & S & M & M & M & B \\
\hline N & P & PS & Z & N & N & N & N \\
S & S & & & S & S & M & M \\
\hline Z & P & P & PS & Z & N & N & N \\
& M & M & & & S & M & M \\
\hline PS & P & P & P & PS & Z & N & N \\
& M & M & M & & & S & S \\
\hline P & P & P & P & PS & PS & Z & N \\
M & B & B & M & & & & S \\
\hline P & P & P & P & P & PS & Z & Z \\
B & B & B & M & M & & & \\
\hline
\end{tabular}

Table 2

\begin{tabular}{|l|l|l|l|l|l|l|l|}
\hline \multirow{2}{*}{ E } & \multicolumn{7}{|l}{ EC } \\
\cline { 2 - 8 } & N & N & N & Z & P & P & P \\
& B & M & S & & S & M & B \\
\hline N & N & N & N & N & N & Z & Z \\
B & B & B & M & M & S & & \\
\hline N & N & N & N & N & N & Z & Z \\
M & B & B & M & S & S & & \\
\hline N & N & N & N & N & Z & P & PS \\
S & B & M & S & S & & S & \\
\hline Z & N & N & N & Z & P & P & P \\
& M & M & S & & S & M & M \\
\hline P & N & N & Z & P & P & P & P \\
S & M & S & & S & S & M & B \\
\hline P & Z & Z & P & P & P & P & P \\
M & & & S & S & M & B & B \\
\hline P & Z & Z & P & P & P & P & P \\
B & & & S & M & M & B & B \\
\hline
\end{tabular}

Table 3

\begin{tabular}{|l|l|l|l|l|l|l|l|}
\hline \multirow{2}{*}{ E } & \multicolumn{6}{|l|}{ EC } \\
\cline { 2 - 8 } & N & N & N & Z & P & P & P \\
& B & M & S & & S & M & B \\
\hline N & P & N & N & N & N & N & P \\
B & S & S & B & B & B & M & S \\
\hline N & P & N & N & N & N & N & Z \\
M & S & S & B & M & M & S & \\
\hline N & Z & N & N & N & N & N & Z \\
S & & S & M & M & S & S & \\
\hline Z & Z & N & N & N & N & N & Z \\
& & S & S & S & S & S & \\
\hline P & Z & Z & Z & Z & Z & Z & Z \\
S & & & & & & & \\
\hline P & P & N & P & P & P & P & P \\
M & B & S & S & S & S & S & B \\
\hline P & P & P & P & P & P & P & P \\
B & B & M & M & M & S & S & B \\
\hline
\end{tabular}

\section{Defuzzification}

In this paper, the gravity method is adopted to eliminate the influence of the fuzzy processing ${ }^{[10]}$. By using the fuzzy control rule of table 1、 table 2 and table 3, the output of the fuzzy controller can be got on the basis of gear's positional deviation and its rate of change. In this passage is taken as an example to introduce how to eliminate the influence of the fuzzy processing.

$$
\begin{aligned}
& \mathrm{K}_{\mathrm{P}}=\mathrm{K}_{\mathrm{P} 0}+\Delta \mathrm{K}_{\mathrm{P}} \\
& \mathrm{K}_{\mathrm{I}}=\mathrm{K}_{\mathrm{I} 0}+\Delta \mathrm{K}_{\mathrm{P}} \\
& \mathrm{K}_{\mathrm{D}}=\mathrm{K}_{\mathrm{D} 0}+\Delta \mathrm{K}_{\mathrm{D}}
\end{aligned}
$$

In the Eq.2 and are initial value of the location PID controller, namely scaling factor 、 integral coefficient and differential coefficient; and are the corrections of scaling factor vintegral coefficient and differential coefficient; and are the revised positional PID controls parameter.

\section{Simulation results}

In order to verify the control performance of the designed controller of gear shifting actuator based on fuzzy adaptation and double-closed-loop PID, the paper used the Matlab/Simulink software to 
establish a fuzzy dual closed-loop PID controller model including the DC motor, and simulated its control effect. In the simulating model, the parameters of the shifting motor and PID controller, membership function of the fuzzy controller and fuzzy rules were set up to simulate, and then the simulating results were compared with the control effect of traditional PID. The parameters of shifting motor is shown in Table 4.

Table 4. Parameters of shifting motor

\begin{tabular}{|c|c|c|}
\hline Motor parameters & Value & Unit \\
\hline Rated voltage & 12 & $\mathrm{~V}$ \\
\hline $\begin{array}{l}\text { Rated / no-load } \\
\text { current }\end{array}$ & $\begin{array}{l}6.34 / 0.9 \\
5\end{array}$ & \multirow{2}{*}{$\begin{array}{l}\text { A } \\
\text { rpm }\end{array}$} \\
\hline $\begin{array}{l}\text { Rated / no-load } \\
\text { speed }\end{array}$ & $\begin{array}{l}4120 / 47 \\
18\end{array}$ & \\
\hline Rated torque & 127.17 & $\mathrm{mNm}$ \\
\hline $\begin{array}{l}\text { Maximum output } \\
\text { power }\end{array}$ & 122.89 & $\mathrm{w}$ \\
\hline $\begin{array}{l}\text { Armature } \\
\text { resistance } \\
\text { Armature } \\
\text { inductance } \\
\text { Torque constant } \\
\text { Rotor inertia }\end{array}$ & $\begin{array}{l}0.46 \\
0.216 \\
20.06 \\
4.8 \times 10- \\
4\end{array}$ & $\begin{array}{l}\mathrm{mH} \\
\mathrm{mNm} / \mathrm{A} \\
\mathrm{kg} \mathrm{m}^{2}\end{array}$ \\
\hline $\begin{array}{l}\text { Maximum } \\
\text { efficiency }\end{array}$ & 72 & $\%$ \\
\hline
\end{tabular}

Taking shifting to the first gear as an example, Fig 5 is the simulating and contrasting curve of the gear position and motor speed at the process of neutral gear to the first gear.

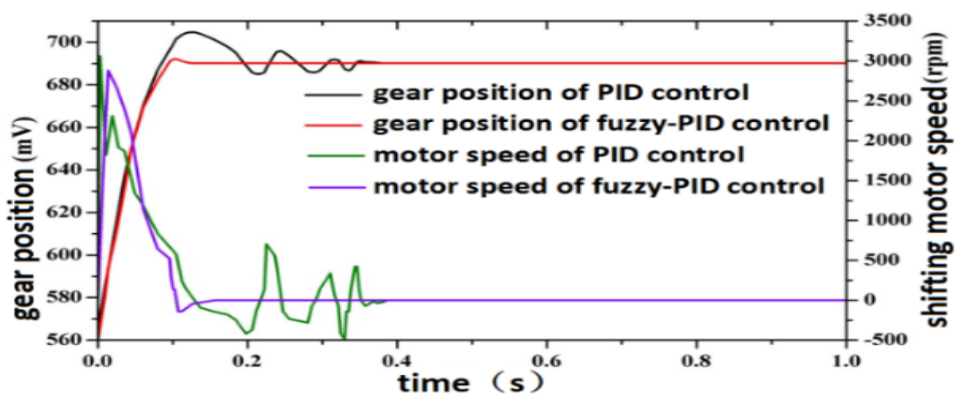

Figure 5. Simulation results of the AMT shifting from neutral gear to the first gear

\section{Conclusions}

From the simulation results, we can get the following conclusions: the designed controller of gear shifting actuator based on fuzzy adaptation and double closed-loop PID has a fast response speed, high control accuracy, and good stability. And the control of each shifting can be completed and reach stable state in $130 \mathrm{~ms}$, which realized the fast and accurate control of the shifting movement, thus can effectively shorten the time of power interruption of AMT system and improve the shifting quality of AMT system.

\section{Acknowledgement}

This work was supported by International S\&T Cooperation Program of China (Grant No. 2014DFA71790), the Key Scientific and Technological Project of Jilin Province (Grant No. 20160519008JH, 20170204073GX) , Chinese National Natural Science Foundation(Grant No. 51575220) and Graduate Innovation Fund of Jilin University（Grant No. 2016170）”. 


\section{References}

[1] FuShui, Z., DongCai, T., Ying, L., ZhaoJin, T., “Analysis on Structure and development of hybrid electric vehicle transmission,” Hunnan Agricultural Machinery.2007(9):28-31.

[2] Dong, P., "Research on the Integrated Control of No_separated_clutch AMT,” Jilin University.

[3]Xuefeng,Z., "Starting Control of AMT Vehicle Based on Estimate of Target Oil Pedal Opening,” Chongqing University

[4] N. Kanagaraj,P. Sivashanmugam,S. Paramasivam. “A Fuzzy Logic Based Supervisory Hierarchical Control Scheme for Real Time Pressure Control” International Journal of Automation \& Computing, 2009, 01:88-96.

[5] Hongche G., Tianyu Q., Jiuchun J., "Design of Fuzzy Controller for Direct Drive Wind Turbines”,Journal of Shanghai Jiaotong University(Science),2012,02:209-214.

[6]Huigui R., Hui Z., Zhengqiang L., Yuxiang X.. “Tuning of fuzzy PID controller for Smith predictor” Journal of Central South University of Technology, 2010, 03:566-571.

[7] Hongxing L. "Relationship between fuzzy controllers and PID controllers", Science in China (Series E: Technological Sciences), 1999, 02:215-224.

[8] Xing, G., Ming,Z., Yu-feng,Y., "position control for sampling arm based on self-adaptng fuzzy PID” Chinese Journal of Engineering Design,2012(05)

[9] Tian, Q., Qi,L., Peng, Y., Yuan,Z., “Motion Control of Omni-directional Robot Based on Fuzzy PID Control,” Electronic Science and Technology,2011(9),page131-133.

[10] Xinyi, R., Fengshan, D., Huagui, H., Shangbin Z,. “Application of Improved Fuzzy Immune PID Controller to Bending Control System,” Journal of Iron and Steel Research (International), 2011, 03:28-33. 